Copyright (C) 2013 IEEE. Personal use of this material is permitted. Permission from IEEE must be obtained for all other uses, in any current or future media, including reprinting/republishing this material for advertising or promotional purposes, creating new collective works, for resale or redistribution to servers or lists, or reuse of any copyrighted component of this work in other works. 


\title{
Robust repeated pole placement
}

\author{
Robert Schmid, Lorenzo Ntogramatzidis, Thang Nguyen and Amit Pandey
}

\begin{abstract}
We consider the classic problem of pole placement by state feedback. Recently [1] offered an eigenstructure assignment algorithm to obtain a novel parametric form for the pole-placing gain matrix to deliver any set of desired closed-loop eigenvalues, with any desired multiplicities. In this paper we employ this parametric formula to introduce an unconstrained nonlinear optimisation algorithm to obtain a gain matrix that delivers any desired pole placement with optimal robustness.
\end{abstract}

\section{INTRODUCTION}

We consider the classic problem of repeated pole placement for linear time-invariant (LTI) systems in state space form

$$
\dot{x}(t)=A x(t)+B u(t)
$$

where, for all $t \in \mathbb{R}, x(t) \in \mathbb{R}^{n}$ is the state and $u(t) \in$ $\mathbb{R}^{m}$ is the control input, and $A$ and $B$ are appropriate dimensional constant matrices. We also assume that $B$ has full column rank, and that the pair $(A, B)$ is reachable. We let $\mathscr{L}=\left\{\lambda_{1}, \ldots, \lambda_{v}\right\}$ be a self-conjugate set of $v \leq n$ complex numbers, with associated algebraic multiplicities $\mathscr{M}=\left\{m_{1}, \ldots, m_{v}\right\}$ satisfying $m_{1}+\cdots+m_{v}=n$. The problem of exact pole placement (EPP) by state feedback is that of finding a real gain matrix $F$ such that the closed-loop matrix $A+B F$ has eigenvalues given by the set $\mathscr{L}$ with multiplicities given by $\mathscr{M}$, i.e., $F$ satisfies the equation

$$
(A+B F) X=X \Lambda
$$

where $\Lambda$ is a $n \times n$ Jordan matrix obtained from the eigenvalues of $\mathscr{L}$, including multiplicities, and $X$ is a matrix of closed-loop eigenvectors of unit length. The matrix $\Lambda$ can be expressed in the Jordan (complex) canonical form

$$
\Lambda=\left[\begin{array}{ccc}
J\left(\lambda_{1}\right) & \cdots & 0 \\
\vdots & \ddots & \vdots \\
0 & \cdots & J\left(\lambda_{v}\right)
\end{array}\right]
$$

where each $J\left(\lambda_{i}\right)$ represents a Jordan matrix for the eigenvalue $\lambda_{i}$ of order $m_{i}$, and may be composed of up to $m$

Robert Schmid is with the Department of Electrical and Electronic Engineering, University of Melbourne, Australia. Lorenzo Ntogramatzidis is with the Department of Mathematics and Statistics, Curtin University, Perth, WA 6848, Australia. Thang Nguyen is with the Department of Engineering, University of Exeter, UK. Amit Pandey is with the Department of Mechanical and Aerospace Engineering, University of California, San Diego, USA. E-mail: rschmid@unimelb.edu.au; L.Ntogramatzidis@curtin.edu.au;

T.Nguyen-Tien@exeter.ac.uk; amitpandey.ent@gmail.com mini-blocks ${ }^{1}$

$$
J\left(\lambda_{i}\right)=\left[\begin{array}{ccc}
J_{1}\left(\lambda_{i}\right) & \cdots & 0 \\
\vdots & \ddots & \vdots \\
0 & \cdots & J_{g_{i}}\left(\lambda_{i}\right)
\end{array}\right]
$$

where $g_{i} \leq m$. We use $\mathscr{P} \triangleq\left\{p_{i, j} \mid 1 \leq i \leq v, 1 \leq j \leq g_{i}\right\}$ to denote the order of each Jordan mini-block $J_{j}\left(\lambda_{i}\right)$, and we assume without loss of generality that for each $i$, these are in descending order $p_{i, 1} \geq p_{i, 2} \geq \cdots \geq p_{i, g_{i}}$. It is well-known that for controllable $(A, B)$, arbitrary multiplicities can be assigned, but the possible orders of the associated Jordan structures are constrained by the system controllability indices (or Kronecker invariants) $\left\{q_{1}, \ldots, q_{m}\right\}$ as follows: [2]

$$
\begin{aligned}
\sum_{i=1}^{v} p_{i, 1} & \geq q_{1} \\
\sum_{i=1}^{v} p_{i, 1}+p_{i, 2} & \geq q_{1}+q_{2} \\
& \vdots \\
\sum_{i=1}^{v} \sum_{j=1}^{m} p_{i, j} & \geq q_{1}+q_{2}+\cdots+q_{m}
\end{aligned}
$$

The last equation assumes $p_{i, j}=0$ if $g_{i}<j \leq m$. If $\mathscr{L}, \mathscr{M}$ and $\mathscr{P}$ satisfy the conditions of the Rosenbrock theorem, we say that $\mathscr{L}, \mathscr{M}$ and $\mathscr{P}$ define an admissible Jordan structure.

In order to consider optimal selections for the gain matrix, it is important to have a parametric formula for the set of gain matrices that deliver the desired pole placement, and numerous such parameterisations have appeared in the literature in the past three decades. Kautsky et al. [3] introduced a parametric form involving a $\mathrm{QR}$-factorisation for matrix $B$ and a Sylvester equation for $X$, but required $\Lambda$ in (2) to be a diagonal matrix. In particular this requires the desired multiplicities to satisfy $m_{i} \leq m$ for all $i \in\{1, \ldots, v\}$. This limitation is inherited by the MATLAB ${ }^{\circledR}$ routine place.m that is based on [3]. The pole-placement methods of Byers and Nash [4] and Tits and Yang [5] similarly employed the parametric form of [3] and likewise cannot assign poles with multiplicity greater than the rank of $B$. Our own recent paper [6] offered a novel parametric form based on Moore's algorithm [7], but this also required $\Lambda$ to be diagonal, and hence also assumed the closed-loop eigenvalues to have multiplicities of at most $m$.

Other parameterisations have been presented in the literature that do not impose a constraint on the multiplicity of the

\footnotetext{
${ }^{1}$ Each $J\left(\lambda_{i}\right)$ is composed of up to $m$ mini-blocks because, as will be mentioned in the sequel, when the pair $(A, B)$ is reachable, the dimension of $\operatorname{ker}\left[\begin{array}{lll}A-\lambda I & B\end{array}\right]$ is equal to $m$ for any $\lambda \in \mathbb{C}$.
} 
eigenvalues to be assigned. Bhattacharyya and de Souza [8] gave a procedure for obtaining the gain matrix by solving a Sylvester equation in terms of an $n \times m$ parameter matrix, provided the closed-loop eigenvalues do not coincide with the open loop ones. Fahmy and O'Reilly in [9] presented a parametric form in terms of the inverses of the matrices $A-\lambda_{i} I_{n}$ (where $I_{n}$ denotes the $n \times n$ identity matrix), which also required the assumption that the closed-loop eigenvalues were all distinct from the open loop ones.

More recently, Chu [10] revisited the parametric formula of [3] for the case where $\Lambda$ was any admissible Jordan matrix, and obtained a parameterisation for the pole placing matrix $F$ by using the eigenvector matrix $X$ as a parameter. Ait Rami et al. [11] also considered the case where $\mathscr{L}$ contained any desired closed-loop eigenvalues and multiplicities, and proposed a parametric form for $F$ in terms of the solution to a Sylvester equation, also using the eigenvector matrix $X$ as a parameter. Thus the parametric forms for $F$ offered in [10] and [11] are the most general currently available in the literature. On the other hand, maximum generality in these parametric formulae has been achieved at the expense of efficiency. Where methods [3]-[9] all employed parameter matrices of dimension $m \times n$, the parameter matrices in [10] and [11] have dimension $n \times n$. In our recent work [1], we gave a parameterisation for the pole-placing feedback that combines the generality of [10] and [11] with the efficiency that comes from an $m \times n$ dimensional parameter matrix. The parametric form for the pole-placing gain matrix $F$ can obtain any desired Jordan structure that satisfies the structural constraints imposed by the Rosenbrock theorem in terms of the controllability indices, without any additional requirement on their multiplicity or non-overlap with the open-loop eigenstructure.

In [1] we employed this novel parametric form to seek the solution to the minimum gain exact pole placement problem (MGEPP), which involves solving the EPP problem and also obtaining the feedback matrix $F$ that has the smallest gain, which in turn minimises the control amplitude or energy required. In this paper we employ the parametric form to consider the robust exact pole placement problem (REPP), which involves obtaining $F$ that solves the EPP problem and also renders the eigenvalues of $A+B F$ as insensitive to perturbations in $A, B$ and $F$ as possible. Numerous results [12], [13], [14] have appeared linking the sensitivity of the eigenvalues to various measures of the conditioning of $X$, the matrix of closed loop eigenvectors. A commonly used measure is the Frobenius condition number of $X$.

For the case of diagonal $\Lambda$, there has been considerable literature on this problem. Papers addressing the conditioning of the eigenvector matrix include [3], [4], [5], [11], [15], and our own earlier work [6]. In this paper we extend our earlier work and address the general problem of robust pole placement for a possibly defective $\Lambda$. We utilise our parametric form for the matrices $X$ and $F$ that solve (2) to introduce an unconstrained nonlinear optimisation problem that seeks the parameter matrix $K$ that minimises the condition number of $X$ with respect to Frobenius norm. Finally we demonstrate the performance of our algorithm by considering an example involving the assignment of deadbeat modes, and compare the performance against the method of [11].

\section{Repeated Pole Placement}

Here we briefly summarise the parametric formula for a gain matrix $F$ that solves the exact pole placement problem, in terms of an arbitrary real parameter matrix that appears in [1]. We begin with some definitions and notation. For each $i \in\{1, \ldots, v\}$, we define the matrix

$$
S\left(\lambda_{i}\right) \triangleq\left[\begin{array}{ll}
A-\lambda_{i} I_{n} & B
\end{array}\right] .
$$

Since each $S\left(\lambda_{i}\right)$ has $n$ rows and $n+m$ columns, and the pair $(A, B)$ is reachable, the dimension of the kernel of $S\left(\lambda_{i}\right)$ is equal to $m$. We denote by $N_{i}$ a basis matrix for the kernel of $S\left(\lambda_{i}\right)$. It follows that, if $\lambda_{i+1}=\bar{\lambda}_{i}$, then $N_{i+1}$ is given by $N_{i+1}=\bar{N}_{i}$.

We let

$$
M_{i} \triangleq\left[\begin{array}{ll}
A-\lambda_{i} I_{n} & B
\end{array}\right]^{\dagger},
$$

where ${ }^{\dagger}$ indicates the Moore-Penrose pseudo-inverse.

For any matrix $X$ we use $X(l)$ to denote the $l$-th column of $X$. If $X$ is a vector or matrix with $n+m$ rows, we define the vectors or matrices $\bar{\pi}\{X\}$ and $\underline{\pi}\{X\}$, obtained by taking the first $n$ and last $m$ rows of $X$, respectively.

Given a set of $v$ self-conjugate complex numbers $\mathscr{L}=$ $\left\{\lambda_{1}, \ldots, \lambda_{v}\right\}$ containing exactly $\sigma$ complex conjugate pairs, we say that $\mathscr{L}$ is $\sigma$-conformably ordered if the first $2 \sigma$ values of $\mathscr{L}$ are complex while the remaining are real, and for all odd $k \leq 2 \sigma$ we have $\lambda_{k+1}=\bar{\lambda}_{k}$. For example, the set $\mathscr{L}=\{10 j,-10 j, 2+2 j, 2-2 j, 7\}$ is 2-conformably ordered. Notice that, since $\mathscr{L}$ is symmetric, we have $m_{i}=$ $m_{i+1}$ for odd $i \leq \sigma$.

Let $\mathscr{L}=\left\{\lambda_{1}, \ldots, \lambda_{v}\right\}$ be $\sigma$-conformably ordered. Let $\mathscr{L}$, $\mathscr{M}$ and $\mathscr{P}$ define an admissible Jordan structure. Let $K \triangleq$ $\operatorname{diag}\left\{K_{1}, \ldots, K_{V}\right\}$, where $K_{i}$ is a real matrix of dimension $m \times m_{i}$, for each $i \geq 2 \sigma, K_{i}$ is a real matrix of dimension $m \times m_{i}$, and for all odd $i \leq 2 \sigma$, we have $K_{i}=\bar{K}_{i+1}$. Further, let each $K_{i}$ matrix be partitioned as

$$
K_{i}=\left[\begin{array}{l|l|l|l}
K_{i, 1} & K_{i, 2} & \ldots & K_{i, g_{i}}
\end{array}\right],
$$

where each $K_{i, k}$ is of dimension $m \times p_{i, k}$. For all odd $i \in\{1, \ldots, 2 \sigma\}$ and for each $i \in\{2 \sigma+1, \ldots, v\}$ and $k \in$ $\left\{1, \ldots, g_{i}\right\}$ we build vector chains of length $p_{i, k}$ as follows:

$$
\begin{aligned}
h_{i, k}(1) & =N_{i} K_{i, k}(1), \\
h_{i, k}(2) & =M_{i} \bar{\pi}\left\{h_{i, k}(1)\right\}+N_{i} K_{i, k}(2), \\
& \vdots \\
h_{i, k}\left(p_{i, k}\right) & =M_{i} \bar{\pi}\left\{h_{i, k}\left(p_{i, k}-1\right)\right\}+N_{i} K_{i, k}\left(p_{i, k}\right) .
\end{aligned}
$$

From these column vectors we construct matrices

$$
H_{i, k} \triangleq\left[h_{i, k}(1)\left|h_{i, k}(2)\right| \ldots \mid h_{i, k}\left(p_{i, k}\right)\right]
$$

of dimension $(n+m) \times p_{i, k}$, and real matrices

$$
H_{i} \triangleq \begin{cases}\mathfrak{R e}\left\{\left[H_{i, 1}|\ldots| H_{i, g_{i}}\right]\right\} & i \in\{1, \ldots, 2 \sigma\} \text { odd } \\ \mathfrak{I m}\left\{\left[H_{i-1,1}|\ldots| H_{i-1, g_{i}}\right]\right\} & i \in\{1, \ldots, 2 \sigma\} \text { even } \\ {\left[H_{i, 1}|\ldots| H_{i, g_{i}}\right]} & i \in\{2 \sigma+1, \ldots, v\}\end{cases}
$$


of dimension $(n+m) \times m_{i}$. Finally, we define

$$
\begin{aligned}
H_{K} & \triangleq\left[H_{1}|\ldots| H_{V}\right] \\
V_{K} & \triangleq \bar{\pi}\left\{H_{K}\right\} \\
W_{K} & \triangleq \underline{\pi}\left\{H_{K}\right\}
\end{aligned}
$$

of dimensions $(n+m) \times n, n \times n$ and $m \times n$, respectively. The dependence upon $K$ of the matrices defined in (15-17) has been made explicit.

The main result of [1] is the following.

Theorem 2.1: [1] For almost all choices of the parameter matrix $K$, matrix $V_{K}$ is invertible, i.e., $V_{K}$ is generically invertible for every choice of $K$ except possibly those laying in a proper algebraic variety. The set of all feedback matrices such that the Jordan structure of $A+B F$ is described by $\mathscr{L}, \mathscr{M}$ and $\mathscr{P}$ is parameterised in $K$ as

$$
F_{K}=W_{K} V_{K}^{-1}
$$

where $V_{K}$ and $W_{K}$ are obtained with a parameter matrix $K$ such that $V_{K}$ is invertible.

The above formulation takes its inspiration from the proof of Proposition 1 in [16], and hence we shall refer to (18) as the Klein-Moore parametric form for $F$. Next we illustrate the procedure of construction of the $V_{K}, W_{K}$ and $F$ in a simple example.

Example 2.1: Consider the reachable pair $(A, B)$ with

$$
A=\left[\begin{array}{lll}
0 & 0 & 0 \\
0 & 3 & 0 \\
0 & 0 & 0
\end{array}\right], \quad B=\left[\begin{array}{ll}
1 & 0 \\
2 & 0 \\
0 & 3
\end{array}\right]
$$

Our aim is to ultimately find a feedback matrix $F$ that assigns the eigenvalue -2 with multiplicity equal to $n=3$, i.e., $\mathscr{L}=\{-2\}$ and $\mathscr{M}=\{3\}$. It is easy to see that the controllability indices of this pair are 2 and 1 . Hence, the Rosenbrock theorem tells us that it is not possible to find a feedback matrix $F$ such that $\Lambda$ has three Jordan blocks relative to the closed-loop eigenvalue -2 . In other words, in this case the closed-loop matrix will be defective, and its only admissible Jordan structures are given by

$$
\left[\begin{array}{cc|c}
-2 & 1 & 0 \\
0 & -2 & 0 \\
\hline 0 & 0 & -2
\end{array}\right] \text { and }\left[\begin{array}{ccc}
-2 & 1 & 0 \\
0 & -2 & 1 \\
0 & 0 & -2
\end{array}\right]
$$

Hence, $\mathscr{L}=\{-2\}$ and $\mathscr{M}=\{3\}$, while $\mathscr{P}=\{2,1\}$ and $\mathscr{P}=\{3\}$ are the admissible Jordan structures. Let us consider first the case $\mathscr{P}=\{2,1\}$. Here, $v=1, i=1, g_{1}=2$, $p_{1,1}=2$ and $p_{1,2}=1$. In order to construct the chains defined above, we compute a basis $N_{1}$ for the null-space of $\left[\begin{array}{lll}A-(-2) I_{n} & B\end{array}\right]$ and $M_{1}=\left[A-(-2) I_{n} B\right]^{\dagger}$. We obtain

$N_{1}=\left[\begin{array}{cc}5 & 0 \\ 4 & 0 \\ 0 & -3 \\ -10 & 0 \\ 0 & 2\end{array}\right], \quad M_{1}=\left[\begin{array}{ccc}\frac{58}{141} & -\frac{4}{141} & 0 \\ -\frac{10}{141} & \frac{25}{141} & 0 \\ 0 & 0 & \frac{2}{13} \\ \frac{25}{141} & \frac{8}{141} & 0 \\ 0 & 0 & \frac{3}{13}\end{array}\right]$.

Since $v=1$ and $g_{1}=1$, we have $K=K_{1}=\left[K_{1,1} K_{1,2}\right]$, where $K_{1,1}$ is $m \times p_{1,1}=2 \times 2$ and $K_{1,2}$ is $m \times p_{1,2}=2 \times 1$. Let us choose for example the parameter matrices

$$
K_{1,1}=\left[\begin{array}{c|c}
1 & 3 \\
-1 & -1
\end{array}\right] \text { and } K_{1,2}=\left[\begin{array}{l}
2 \\
1
\end{array}\right]
$$

With this choice we find

$$
\begin{aligned}
& h_{1,1}(1)=N_{1}\left[\begin{array}{c}
1 \\
-1
\end{array}\right]=\left[\begin{array}{lll|ll}
5 & 4 & 3 & -10 & 2
\end{array}\right]^{\mathrm{T}} \\
& h_{1,1}(2)=M_{1}\left[\begin{array}{l}
5 \\
4 \\
3
\end{array}\right]+N_{1}\left[\begin{array}{c}
3 \\
-1
\end{array}\right]
\end{aligned}
$$

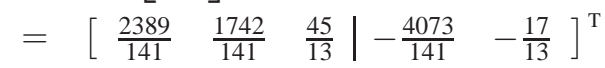

$$
\begin{aligned}
& h_{1,2}(1)=N_{1}\left[\begin{array}{l}
2 \\
1
\end{array}\right]=\left[\begin{array}{lll|ll}
10 & 8 & -3 & -20 & 2
\end{array}\right]^{\mathrm{T}} \text {. }
\end{aligned}
$$

Now, we define $H_{1,1}=\left[h_{1,1}(1) h_{1,1}(2)\right]$ whose size is $(n+$ $m) \times p_{1,1}=5 \times 2$, and $H_{1,2}=\left[h_{1,2}(1)\right]$ whose size is $(n+$ $m) \times p_{1,2}=5 \times 1$. Thus, $H=H_{1}=\left[H_{1,1} H_{1,2}\right]$ is $(n+m) \times$ $m_{i}=5 \times 3$. Finally, using (16)-(17) we compute

$V_{K}=\left[\begin{array}{ccc}5 & \frac{2389}{14} & 10 \\ 4 & \frac{1742}{141} & 8 \\ 3 & \frac{45}{13} & -3\end{array}\right], \quad W_{K}=\left[\begin{array}{ccc}-10 & -\frac{4073}{141} & -20 \\ 2 & -\frac{17}{13} & 2\end{array}\right]$.

Notice that with this choice of the parameter matrix $K=$ $K_{1}=\left[\begin{array}{ll}K_{1,1} & K_{1,2}\end{array}\right]$ the square matrix $V_{K}$ is invertible. Indeed, as we will show in Theorem 2.1, (i) for almost all choices (in a Lebesgue measure sense) of the parameter matrix $K$, the square matrix $V_{K}$ is invertible. Moreover, (ii) for such $K$, a feedback matrix that assigns the desired closed-loop eigenstructure is given by $W_{K} V_{K}^{-1}$. Finally, (iii) all feedback matrices yielding the desired closed-loop eigenstructure can be computed as the product $W_{K} V_{K}^{-1}$ for a suitable parameter matrix $K$ such that $V_{K}$ is invertible. Indeed, it is easily verified that the feedback matrix

$$
F_{K}=W_{K} V_{K}^{-1}=\frac{1}{6}\left[\begin{array}{ccc}
8 & -25 & 0 \\
4 & -5 & -4
\end{array}\right]
$$

yields a closed-loop matrix $A+B F_{K}$ whose Jordan structure is given by diag $\left\{\left[\begin{array}{cc}-2 & 1 \\ 0 & -2\end{array}\right],-2\right\}$ as required.

We conclude this example by showing that a single Jordan mini-block of size 3 is also possible. In this case, $K=K_{1}=$ $\left[K_{1,1}\right]$ and $p_{1,1}=3$. Let us choose for example

$$
K_{1,1}=\left[\begin{array}{l|l|l}
1 & 0 & 1 \\
2 & 1 & 0
\end{array}\right] \text {. }
$$

The Jordan chain is built as

$$
\begin{aligned}
& h_{1,1}(1)=N_{1} K_{1,1}, \\
& h_{1,1}(2)=M_{1} \bar{\pi}\left\{h_{1,1}(1)\right\}+N_{1} K_{1,1}(2), \\
& h_{1,1}(3)=M_{1} \bar{\pi}\left\{h_{1,1}(2)\right\}+N_{1} K_{1,1}(3),
\end{aligned}
$$

and $H_{1,1}=\left[h_{1,1}(1) h_{1,1}(2) h_{1,1}(3)\right]$ whose size is $(n+m) \times$ $p_{1,1}=5 \times 3$. Thus, $H=H_{1}=\left[H_{1,1}\right]$, which leads to

$V_{K}=\left[\begin{array}{ccc}5 & \frac{274}{141} & \frac{115097}{141^{2}} \\ 4 & \frac{50}{141} & -\frac{78034}{141^{2}} \\ -6 & -\frac{51}{13} & -\frac{102}{13^{2}}\end{array}\right], W_{K}=\left[\begin{array}{ccc}-10 & \frac{157}{141} & -\frac{191560}{141^{2}} \\ 4 & \frac{8}{13} & -\frac{153}{13^{2}}\end{array}\right]$.

Computing $F_{K}$ as $W_{K} V_{K}^{-1}$ delivers a defective closed-loop matrix which is similar to $\left[\begin{array}{ccc}-2 & 1 & 0 \\ 0 & -2 & 1 \\ 0 & 0 & -2\end{array}\right]$. 


\section{RoBUSt OPTIMAL POLE PLACEMENT}

We firstly note some classic results on eigenvalue sensitivity.

Theorem 3.1: [12, Theorem 4.4.2]

Let $A$ and $X$ be such that $A=X J X^{-1}$, where $J$ is the Jordan form of $A$, and let $A^{\prime}=A+H$. then for each eigenvalue of $A^{\prime}$, there exists an eigenvalue $\lambda$ of $A$ such that

(a) If $A$ is diagonalisable, then

$$
\left|\lambda-\lambda^{\prime}\right| \leq \kappa_{2}(X)\|H\|_{2}
$$

(b) If $A$ is defective, then

$$
\frac{\left|\lambda-\lambda^{\prime}\right|}{\left(1+\left|\lambda-\lambda^{\prime}\right|\right)^{l-1}} \leq \kappa_{2}(X)\|H\|_{2}
$$

where $\kappa_{2}(X):=\|X\|_{2}\left\|X^{-1}\right\|_{2}$ is the spectral condition number of $X$, and $l$ is the size of the largest Jordan mini-block associated with $\lambda$.

Result (a) is known as the Bauer-Fike Theorem. Both results indicate that the condition number of the matrix $X$ may be used a measure of the eigenvalue sensitivity of the matrix $A$. Since the spectral condition number $\kappa_{2}(X)$ is nondifferentiable, it is not amenable to optimisation via gradient search methods. The Frobenius condition number $\kappa_{\text {fro }}(X)=$ $\|X\|_{\text {fro }}\left\|X^{-1}\right\|_{\text {fro }}$ is differentiable, and since $\kappa_{2}(X) \leq \kappa_{\text {fro }}(X)$, many authors, including [4], [11], [15], [18], have used this as their robustness measure. Note it is possible to reduce the Frobenius condition number of a matrix $X$ by suitably scaling the lengths of its column vectors, yet when $X$ is a matrix of eigenvectors, such scaling does not improve the eigenvalue conditioning. Hence when making comparisons of the closed-loop robustness achieved by different control methodologies, we will assume that the column vectors of $X$ have been normalised.

As pointed out in [4], to minimise $\kappa_{\text {fro }}(X)$, for efficient computation we may instead consider the alternative objective function

$$
f(K)=\left\|V_{K}\right\|_{\text {fro }}^{2}+\left\|V_{K}^{-1}\right\|_{\text {fro }}^{2}
$$

with $V_{k}$ as in (16). In order to determine the optimal input parameter matrix $K$ that minimises $f$, we will exploit a gradient search employing the first and second order derivatives of $\left\|V_{K}\right\|_{\text {fro }}^{2}$ and $\left\|V_{K}^{-1}\right\|_{\text {fro }}^{2}$. From these expressions, the gradient and Hessian of $f$ are easily obtained, and unconstrained nonlinear optimisation methods can then be used to seek local minima. Firstly, we define

$$
\Xi_{i} \triangleq \begin{cases}\mathfrak{R e}\left\{K_{i}\right\} & i \in\{1, \ldots, 2 \sigma\} \text { odd, } \\ \mathfrak{I m}\left\{K_{i-1}\right\} & i \in\{1, \ldots, 2 \sigma\} \text { even, } \\ K_{i} & i \in\{2 \sigma+1, \ldots, v\}\end{cases}
$$

Define $\Xi_{i, k}(l, r)$ as the $r$-th entry of $\Xi_{i, k}(l)$. We compute the derivative of $H_{p, q}$ in (14) with respect to $\Xi_{i, k}$. We have

$$
\frac{\partial H_{p, q}}{\partial \Xi_{i, k}(l, r)}=0
$$

for $p \in\{1, \ldots, 2 \sigma\}$ with $p \neq i, p \neq i+\sigma, p+\sigma \neq i$ and $p \in$ $\{2 \sigma+1, \ldots, v\}$ with $p \neq i$. Define

$$
P(i, l) \triangleq \begin{cases}N_{i} & \text { if } l=0, \\ M_{i} \bar{\pi}\left\{M_{i}\right\}^{l-1} \bar{\pi}\left\{N_{i}\right\} & \text { if } l \geq 1, \\ 0 & \text { otherwise. }\end{cases}
$$

For each $i \in\{1, \ldots, \sigma\}, k \in\left\{1, \ldots, g_{i}\right\}, h, l \in\left\{1, \ldots, p_{i, k}\right\}$ and $r \in\{1, \ldots, m\}$ we find

$$
\begin{gathered}
\frac{\partial \mathfrak{R e}\left\{H_{i, k}(h)\right\}}{\partial \Xi_{i, k}(l, r)}=\mathfrak{R e}\{P(i, h-l)\}(r), \\
\frac{\partial \mathfrak{I m}\left\{H_{i, k}(h)\right\}}{\partial \Xi_{i, k}(l, r)}=\mathfrak{I m}\{P(i, h-l)\}(r), \\
\frac{\partial \mathfrak{R e}\left\{H_{i, k}(h)\right\}}{\partial \Xi_{i+\sigma, k}(l, r)}=-\mathfrak{I m}\{P(i, h-l)\}(r), \\
\frac{\partial \mathfrak{I m}\left\{H_{i, k}(h)\right\}}{\partial \Xi_{i+\sigma, k}(l, r)}=\mathfrak{R e}\{P(i, h-l)\}(r) .
\end{gathered}
$$

For each $i \in\{2 \sigma+1, \ldots, v\}, k \in\left\{1, \ldots, g_{i}\right\}, \quad h, l \in$ $\left\{1, \ldots, p_{i, k}\right\}$ and $r \in\{1, \ldots, m\}$ we have

$$
\frac{\partial H_{i, k}(h)}{\partial \Xi_{i, k}(l, r)}=P(i, h-l)(r) .
$$

Let $V_{K}$ and $W_{K}$ be given by (16) and (17) respectively, and let $U_{K}:=V_{K}^{-1}$. Then

$$
\begin{aligned}
& \frac{\partial V_{K}}{\partial \Xi_{i, k}(l, r)}=\bar{\pi}\left\{\frac{\partial H}{\partial \Xi_{i, k}(l, r)}\right\}, \\
& \frac{\partial W_{K}}{\partial \Xi_{i, k}(l, r)}=\underline{\pi}\left\{\frac{\partial H}{\partial \Xi_{i, k}(l, r)}\right\} .
\end{aligned}
$$

The derivatives of $\left\|V_{K}\right\|_{\text {fro }}^{2}$ and $\left\|U_{K}\right\|_{\text {fro }}^{2}$ are given as

$$
\begin{aligned}
& \frac{\partial\left\|V_{K}\right\|_{\text {fro }}^{2}}{\partial \Xi_{i, k}(l, r)}=2 \operatorname{trace}\left(V_{K}^{\mathrm{T}} \frac{\partial V_{K}}{\partial \Xi_{i, k}(l, r)}\right), \\
& \frac{\partial^{2}\left\|V_{K}\right\|_{f r o}^{2}}{\partial \Xi_{i_{1}, k_{1}}\left(l_{1}, r_{1}\right) \partial \Xi_{i_{2}, k_{2}}\left(l_{2}, r_{2}\right)} \\
& \quad=2 \operatorname{trace}\left(\frac{\partial V_{K}^{\mathrm{T}}}{\partial \Xi_{i_{1}, k_{1}}\left(l_{1}, r_{1}\right)} \frac{\partial V_{K}}{\partial \Xi i_{2}, k_{2}\left(l_{2}, r_{2}\right)}\right)
\end{aligned}
$$

Using the well-known formula $\frac{\partial U_{K}}{\partial \Xi_{i, k}(l, r)}=-U_{K} \frac{\partial V_{K}}{\partial \Xi_{i, k}(l, r)} U_{K}$, we compute

$$
\frac{\partial\left\|U_{K}\right\|_{f r o}^{2}}{\partial \Xi_{i, k}(l, r)}=2 \operatorname{trace}\left(-U_{K}^{\mathrm{T}} U_{K} \frac{\partial V_{K}}{\partial \Xi_{i, k}(l, r)} U_{K}\right)
$$

and

$$
\begin{aligned}
& \frac{\partial^{2}\left\|U_{K}\right\|_{\text {fro }}^{2}}{\partial \Xi_{i_{1}, k_{1}}\left(l_{1}, r_{1}\right) \partial \Xi_{i_{2}, k_{2}}\left(l_{2}, r_{2}\right)} \\
& =2 \operatorname{trace}\left(U_{K}^{\mathrm{T}} \frac{\partial V_{K}^{\mathrm{T}}}{\partial \Xi_{i_{2}, k_{2}}\left(l_{2}, r_{2}\right)} U_{K}^{\mathrm{T}} U_{K} \frac{\partial V_{K}}{\partial \Xi_{i_{1}, k_{1}}\left(l_{1}, r_{1}\right)} U_{K}\right. \\
& +U_{K}^{\mathrm{T}} U_{K} \frac{\partial V_{K}}{\partial \Xi_{i_{2}, k_{2}}\left(l_{2}, r_{2}\right)} U_{K} \frac{\partial V_{K}}{\partial \Xi_{i_{1}, k_{1}}\left(l_{1}, r_{1}\right)} U_{K} \\
& \left.+U_{K}^{\mathrm{T}} U_{K} \frac{\partial V_{K}}{\partial \Xi_{i_{1}, k_{1}}\left(l_{1}, r_{1}\right)} U_{K} \frac{\partial V_{K}}{\partial \Xi_{i_{2}, k_{2}}\left(l_{2}, r_{2}\right)} U_{K}\right) .
\end{aligned}
$$




\section{PERFORMANCE COMPARISON}

In this section, we compare the algorithm presented in this paper with the methods given in [11].

Example 4.1: We consider the Example 8 in the Byers Nash [4] collection of benchmark systems that have been investigated over the years by many authors [5], [15], [10], [11]. We use the state matrices $A$ and $B$ from that system, with $n=4$ states and $m=3$ inputs. Differing from [4], we seek to assign all the closed-loop eigenvalues to zero to obtain a deadbeat response, and thus we have $\mathscr{L}=\{0\}$ and $\mathscr{M}=\{4\}$. The controllability indices are $\{2,1,1\}$ and so we see that this can be achieved with a single Jordan mini-block of dimension two, and two blocks of dimension one. Using the method of [11] to minimise the Frobenius condition number of the matrix of eigenvectors $X$, we obtain

$$
F_{1}=\left[\begin{array}{cccc}
0.0201 & -0.6157 & -0.1026 & 0.0178 \\
5.5108 & -3.7659 & 0.8791 & 2.8245 \\
-0.2685 & 4.5596 & -5.2342 & -0.2367
\end{array}\right]
$$

yielding normalised closed-loop eigenvector matrix $X_{1}$ with $\kappa_{\text {fro }}\left(X_{1}\right)=4.000$ and $\left\|F_{1}\right\|_{\text {fro }}=10.099$. Applying our Method (see [1]), we obtain

$$
F_{2}=\left[\begin{array}{cccc}
0.0201 & -0.3818 & -0.1026 & 0.0178 \\
5.5108 & 0.0005 & 0.8791 & 2.8245 \\
-0.2685 & -0.0182 & -5.2342 & -0.2367
\end{array}\right]
$$

yielding $\kappa_{\text {fro }}\left(X_{2}\right)=4.000$, and $\left\|F_{2}\right\|_{\text {fro }}=8.173$, indicating that our method can achieve the same Frobenius conditioning of the eigenvectors, but with reduced gain.

\section{CONCLUSION}

In our recent paper [1] we introduced a novel parametric form for the gain matrix that solves the classic problem of exact pole placement with any desired eigenstructure. In this paper we employed the parametric form to offer a method for obtaining a robust eigenstructure. The effectiveness of the method was compared against a recent alternative method from the literature and shown to achieve equivalent robust conditioning, but with reduced matrix gain. Directions for future research include investigations on the problem of nonovershooting, non-undershooting and monotonic tracking control [19]-[22] with repeated pole placement of the closedloop spectrum.

\section{REFERENCES}

[1] R. Schmid, L. Ntogramatzidis, T. Nguyen and A. Pandey, Repeated pole placement by state feedback with minimum gain, accepted for 21st Mediterranean Conference on Control and Automation, Crete, June 2013

[2] H. H. Rosenbrock, State-Space and Multivariable Theory. New York: Wiley, 1970

[3] J. Kautsky, J. N.K. Nichols and P. Van Dooren, Robust Pole Assignment in Linear State Feedback, International Journal of Control, vol. 41, pp. 1129-1155, 1985.

[4] R. Byers and S. G. Nash, Approaches to robust pole assignment, International Journal of Control, vol. 49, pp. 97-117, 1989.

[5] A. L. Tits and Y. Yang, Globally Convergent Algorithms for Robust Pole Assignment by State Feedback, IEEE Transactions on Automatic Control., vol. 41(10), pp. 1432-1452, 1996.
[6] R. Schmid, T. Nguyen and A. Pandey, Optimal Pole placement with Moore's algorithm, in Proceedings 1st IEEE Australian Control Conference (AUCC 2011), Melbourne, Australia, 2011.

[7] B.C. Moore, On the Flexibility Offered by State Feedback in Multivariable systems Beyond Closed Loop Eigenvalue Assignment, IEEE Transactions on Automatic Control, vol. 21(5), pp. 689-692, 1976.

[8] S.P. Bhattacharyya and E. de Souza, Pole assignment via Sylvester equation, Systems \& Control Letters, vol. 1(4), pp. 261-263, 1981.

[9] M.M. Fahmy and J. O'Reilly, Eigenstructure Assignment in Linear Multivariable Systems-A Parametric Solution, IEEE Transactions on Automatic Control, vol. 28, pp. 990-994, 1983.

[10] E. Chu, Pole assignment via the Schur form, Systems \& Control Letters vol. 56, pp, 303-314, 2007.

[11] M. Ait Rami, S.E. Faiz, A. Benzaouia, and F. Tadeo, Robust Exact Pole Placement via an LMI-Based Algorithm, IEEE Transactions on Automatic Control, Vol. 54(2) pp. 394-398, 2009.

[12] F. Chatelin, Eigenvalues of Matrices, John Wiley and Sons, 1993.

[13] G. Golub, and C.F. van Loan, Matrix Computations, Johns Hopkins University Press, 1983.

[14] G.W. Stewart and J.G. Sun, Matrix Perturbation Theory, Academic press, 1990

[15] A. Varga, Robust Pole Assignment via Sylvester Equation Based State Feedback Parametrization, Proceedings IEEE International Symposium on Computer-Aided Control System Design, Anchorage, USA, 2000.

[16] G. Klein and B.C. Moore, Eigenvalue-Generalized Eigenvector Assignment with State Feedback, IEEE Transactions on Automatic Control, vol. 22(1), pp. 141-142, 1977.

[17] P.J. Antsaklis and Z. Gao, Polynomial and rational interpolation: theory and control applications, International Journal of Control, vol. 58(2), pp. 349-404, 1993.

[18] H.K. Tam and J. Lam, Newton's approach to gain-controlled robust pole placement, IEE Proc.-Control Theory Applications. Vol. 144(5), pp. 439-446, 1997.

[19] R. Schmid, and L. Ntogramatzidis, On the design of non-overshooting linear tracking controllers for right invertible systems. In Proceedings of the $48^{\text {th }}$ Conference on Decision and Control (CDC 09), Shanghai, P.R. China, Dec. 16-18, 2009.

[20] R. Schmid, and L. Ntogramatzidis, Achieving a nonovershooting transient response with multivariable dynamic output feedback tracking controllers. In Proceedings of the $48^{\text {th }}$ Conference on Decision and Control (CDC 09), Shanghai, P.R. China, Dec. 16-18, 2009.

[21] R. Schmid, and L. Ntogramatzidis, A unified method for the design of nonovershooting linear multivariable state-feedback tracking controllers. Automatica, 46: 312-321, 2010.

[22] R. Schmid, and L. Ntogramatzidis, "The design of nonovershooting and nonundershooting multivariable state feedback tracking controllers". Systems \& Control Letters, 61:714-722, 2012. 